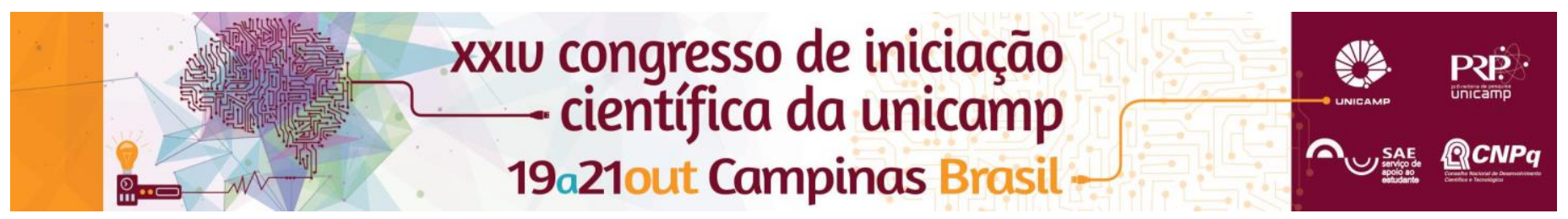

\title{
Estudo de uma nova e promissora classe de fármacos para o tratamento da doença de Chagas
}

\author{
Andressa Bruscato*, Cleiton Moreira da Silva, Ângelo de Fátima, Fernanda Ramos Gadelha
}

\begin{abstract}
Resumo
O Trypanosoma cruzi é o agente etiológico da doença de Chagas (DC) que atinge atualmente entre 16 a 18 milhões de indivíduos, na América Latina. Não há vacina e os tratamentos disponíveis além de gerarem efeitos colaterais diversos têm eficácia limitada. A busca por uma terapia mais específica e, portanto menos tóxica para o hospedeiro vertebrado é uma prioridade. O objetivo deste trabalho foi testar o efeito da imina CMS33 no metabolismo oxidativo, em cepas epimastigotas de $\mathrm{T}$. cruzi. Após tratamento $(4,4 \mu \mathrm{g} / \mathrm{ml})$, os seguintes parâmetros bioquímicos foram analisados: consumo de oxigênio e liberação de $\mathrm{H} 2 \mathrm{O} 2$ e superóxido. Observou-se uma diminuição no consumo de oxigênio e o controle respiratório não apresentou diferenças significativas em relação ao controle. As células tratadas apresentaram aumento na produção de espécies reativas a oxigênio (EROs), sugerindo que o modo de ação dessa droga deve ocorrer por meio de geração de uma situação de estresse oxidativo.
\end{abstract}

\section{Palavras-chave:}

Trypanosoma cruzi, doença de Chagas, bioenergética

\section{A DC, cujo agente etiológico é o} Trypanosoma cruzi atinge atualmente, na América Latina, 16 a 18 milhões de indivíduos. Não há vacina para o seu tratamento e os fármacos disponíveis, além de gerarem efeitos colaterais diversos, têm eficácia limitada. Nesse contexto, é necessária a busca por novos fármacos mais específicos e seletivos, sendo, consequentemente menos tóxicos ao hospedeiro vertebrado. Sob o ponto de vista da estrutura química do benznidazol e nifurtimox, fármacos utilizados no tratamento da doença, a alteração molecular da mesma pode fornecer novas substâncias com um perfil farmacodinâmico e farmacocinético que resulte no aumento da atividade anti-T.cruzi. As iminas constituem uma classe de substâncias orgânicas que demonstram grande variedade de atividades biológicas, porém a sua ação anti-T. cruzi ainda não foi explorada. Há uma série de vantagens sob o ponto de vista de preparação (obtidas em etapa única de síntese), estrutura química (a preparação permite o acesso a uma grande diversidade estrutural de substâncias), permitindo uma posição de destaque, em relação aos fármacos supracitados, nos processos de preparação (escala de produção em quilogramas). Desse modo, o principal objetivo do presente trabalho foi avaliar o efeito da imina CMS33 no metabolismo oxidativo de cepas epimastigotas de T.cruzi.

\section{Resultados e Discussão}

Células $\left(10^{6} \mathrm{cels} / \mathrm{mL}\right)$, cepa Tulahuen 2 de T. cruzi, tratadas com CMS33, na concentração $4.4 \mu \mathrm{g} / \mathrm{ml}$, diminuíram em $75 \%$ o consumo de oxigênio, em relação ao controle não tratado. Após tratamento, como não houve diferença significativa entre os estados 3 e 4, concluiu-se que estas células encontravam-se desacopladas. As células tratadas não apresentaram diferença significativa no controle respiratório em relação ao controle. A produção de EROs, que não é detectada nas células controle, foi expressivamente elevada nas células tratadas.

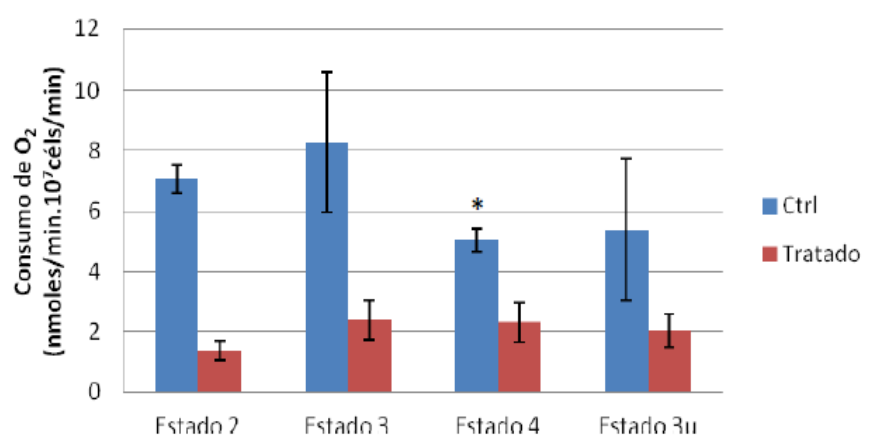

Figura 1. Consumo de $\mathrm{O}_{2}$ da cepa Tulahuen 2 após tratamento com a droga Análise estatística:* teste $t$, onde p> 0.05 , foi considerado não significativo nos grupos indicados.

Tabela 1: Efeito da CMS33 na produção de EROs

\begin{tabular}{|c|c|c|c|}
\hline & \multicolumn{2}{|c|}{$\begin{array}{c}\text { Liberação de } \mathrm{H}_{2} \mathrm{O}_{2} \\
\text { (pmoles } / \mathrm{min}^{-1} \cdot 10^{7} \text { céls) }\end{array}$} & $\begin{array}{c}\text { Produção de } 02^{-} \\
\text {(oxMitosox } / \mathrm{min} .10^{8} \text { céls) }\end{array}$ \\
\hline & Sem Antimicina A & Com Antimicina A & \\
\hline Controle & ND & ND & ND \\
\hline Tratada & $4,96 \pm 1,67^{*}$ & $5,41 \pm 0,26^{*}$ & $0,89 \pm 0,28^{*}$ \\
\hline
\end{tabular}

\section{Conclusões}

Com base nos resultados obtidos, a imina CSM33 mostrou-se promissora para o tratamento do agente etiológico da doença Chagas, uma vez que interfere nos mecanismos bioenergéticos do parasita.

\section{Agradecimentos}

Instituição de fomento: CNPq.

Coura, J.R., and Dias, J.C.P. (2009). Epidemiology, control and surveillance of Chagas disease- 100 years after its discovery. Mem. Inst. Oswaldo Cruz, Rio de Janeiro, 104, 31-40.

Urbina, J.A; and Docampo, R. (2003). Specific chemotherapy of Chagas disease: controversies and advances. Trends Parasitol. 19, 495-501. 